\title{
Forces between electric charges in motion: Rutherford scattering, circular Keplerian orbits, action-at-a-distance and Newton's third law in relativistic classical electrodynamics
}

\author{
J.H.Field \\ Département de Physique Nucléaire et Corpusculaire Université de Genève . 24, quai \\ Ernest-Ansermet CH-1211 Genève 4. \\ e-mail; john.field@cern.ch
}

\begin{abstract}
Standard formulae of classical electromagnetism for the forces between electric charges in motion derived from retarded potentials are compared with those obtained from a recently developed relativistic classical electrodynamic theory with an instantaneous inter-charge force. Problems discussed include small angle Rutherford scattering, Jackson's recent 'torque paradox' and circular Keplerian orbits. Results consistent with special relativity are obtained only with an instantaneous interaction. The impossiblity of stable circular motion with retarded fields in either classical electromagnetism or Newtonian gravitation is demonstrated.
\end{abstract}

Keywords; Special Relativity, Classical Electrodynamics.

PACS 03.50.De 03.30.+p 


\section{Introduction}

Classical electromagnetism (CEM), in particular Maxwell's equations, played an important role in the development of special relativity (SR). This does not however mean that CEM, even as presented in modern text books, is a theory fully compatible with SR and and devoid of interpretational problems. This point was strongly emphasised by Feynman [1]. On the other hand, the most successful physical theory of the 20th century, as much for its powers of prediction as for its precise experimental verification, is quantum electrodynamics (QED) [2] . Again, as stressed by Feynman [3], the elements of this theory are very simple: charged particles and real or virtual photons described by appropriate wavefunctions or propagators and a single coupling constant, the elementary electric charge. In QED the mechanical forces between charges are mediated by the exchange of space-like virtual photons. As discussed, in detail, in a recent paper by the present author [4], the exchange of such photons is instantaneous in the centre-of-mass (CM) frame of two interacting charges. This is a consequence of the relativistic formula for velocity in terms of kinematical quantities: $v=p c^{2} / E$. Energy-momentum conservation shows that $\mathrm{E}\left(\gamma^{*}\right)$ vanishes whereas, in general, $\mathrm{p}\left(\gamma^{*}\right)$ is non-zero for a virtual photon, $\gamma^{*}$, in the CM frame of any scattering process, implying that its velocity is infinite. This conclusion is confirmed by the study, in [4], of the invariant amplitude in momentum space for Møller scattering, $e^{-} e^{-} \rightarrow e^{-} e^{-}$, and its Fourier transform. Recently, convincing experimental evidence has been obtained $[5,6]$

for the non-retarded nature of 'bound' magnetic fields with $r^{-2}$ dependence, (associated in QED with virtual photon exchange) in a modern version, probing small $r$ values, of the Hertz experiment [7] in which the electromagnetic waves associated with the propagation of real photons (fields with $r^{-1}$ dependence) were originally discovered.

There is no reason to suppose that the space-like virtual photons responsible for the electromagnetic forces in Møller scattering should not also be at the origin of the electromagnetic forces between the electrons in two current-carrying conductors separated by macroscopic distances. Thus there should be a close connection between CEM and QED. Just this connection was explored in the second part of [4]. In the first part of this paper all of the mechanical equations of CEM, as well as Maxwell's equations, were derived from Coulomb's inverse-square force law, SR and Hamilton's Principle. The equations describing inter-charge forces, although consistent with standard CEM formulae in the limit where $\mathrm{O}\left(\beta^{2}\right)$ and higher correction terms are neglected, differ significantly from them when such terms are included. The main aim of the present paper is a comparison of the RCED (standing for Relativistic Classical Electro-Dynamics) formulae from [4] with the conventional text book formulae of CEM, to be found, for example, in $[8,9,10]$. Indeed it is found that the latter, corresponding, for moving charges, to retarded potentials and fields do not respect the constraints of SR. As will be seen, this is one source of the previously unresolved problems of CEM which Feynman found to be of such great interest.

The other source of problems and paradoxes in CEM is that electromagnetic forces apparently do not respect Newton's third law. An explanation in terms of non-mechanical momentum and angular momentum residing in static electric and magnetic fields has been 
attempted by many authors $[11,12,13,14,15,16,17]$ over the last half-century. The present author's opinion is that this is not the correct explanation, and that Newton's third law is actually always respected by electromagnetic forces, without the need to introduce non-mechanical degrees of freedom. One important point in this connection is the instantaneous nature of electromagnetic forces, a second is the correct treatment of relativistic effects, and the third is the understanding that the mass of an object is only a constant in the complete absence of interactions, which is evidently not the case for objects undergoing mutual electromagnetic interactions ${ }^{1}$.

In fact it is clear that when all physical quantities are properly defined Newton's third law must be obeyed because it is a necessary consequence of Newton's first law, (the law of inertia) and the definition of 'force' provided by the relativistic generalisation of Newton's second law. The proof of this is now briefly sketched. Given a dynamical theory it is always possible to obtain an equation such as:

$$
\dot{\vec{p}}_{O}=\vec{f}_{O}\left(X, \alpha_{i}, \alpha_{j} \ldots\right)
$$

where $\vec{p}_{O}$ is the relativistic momentum (see (1.6) below) of an object O, the dot denotes a time derivative and $\overrightarrow{f_{O}}$ is a known function of the space-time position $X \equiv(c t ; \vec{x})$ of $\mathrm{O}$ and some fixed parameters $\alpha_{i}, \alpha_{j} \ldots$. The vector $\vec{f}_{O}$ is the relativistic generalisation of what is defined by Newton's second law as a 'force'. Actually, however, this naming operation is the entire physical and logical content of the second law. The dynamics is completely defined by the knowledge of the time derivative of the relativistic momentum (1.1). Nothing is gained, except in an anthropomorphic sense, by baptising the right side of this equation a 'force'. If it happens that $\vec{f}_{O}=0$ in (1.1) then:

$$
\vec{p}_{O}=\text { constant }
$$

which is Newton's first law. Suppose now $\mathrm{O}$ is composed of two parts $\mathrm{O}_{1}$ and $\mathrm{O}_{2}$ so that:

$$
\vec{p}_{O}=\vec{p}_{O_{1}}+\vec{p}_{O_{2}}=\text { constant }
$$

Differentiating (1.3) with respect to time and transposing gives:

$$
\dot{\vec{p}}_{O_{1}}=-\dot{\vec{p}}_{O_{2}}
$$

or, using (1.1)

$$
\vec{f}_{O_{1}}=-\vec{f}_{O_{2}}
$$

which is Newton's third law. It is clear that it is a necessary consequence of the first law (1.2) and the definition (1.1) of 'force' provided by the second law. The third law must then be obeyed if the relativistic momentum is correctly defined.

The general definition of the relativistic momentum of an object is:

$$
\vec{p} \equiv m(X) \frac{d X}{d \tau}=m(X) \gamma \vec{v}
$$

\footnotetext{
${ }^{1}$ The concept of an effective mass for a particle, depending upon the strength of the interactions it undergoes, is a familiar one in the theory of conduction in the solid state. The relation between the energy and momentum of a conduction electron can be markedly different from that for a free electron, for those occupying energy levels near to the upper or lower boundaries of a conduction band [18].
} 
where $\vec{v}$ is the object's velocity, $\tau$ its proper time, $\gamma \equiv 1 / \sqrt{1-\beta^{2}}, \beta \equiv v / c$ and $X$ is the space-time position of the object. Only if the latter is in free space, and undergoes no interaction with the force fields of other objects, is the mass, $m$, a constant. A simple example of the variability of the masses of interacting objects is provided by the case of two equally massive, equally charged, objects moving towards each other along a straight line in their common $\mathrm{CM}$ frame. At a certain distance, $x_{O}$, of each object from their common center of energy, they come to rest, due to their mutual repulsion. The energy of the system in the CM system (i.e. its mass times $c^{2}$ ) is then:

$$
E=2 m c^{2}+\frac{q^{2}}{2 x_{O}} \equiv 2 m\left(x_{O}\right) c^{2}
$$

where $q$ is the charge of each object and $m$ its mass in the absence of interactions. The definition of the spatially dependent mass in the last member of (1.7) follows from the symmetry of the problem -a system at rest composed of two equal sub-systems at rest. Thus:

$$
m\left(x_{O}\right)=m+\frac{q^{2}}{4 c^{2} x_{O}}
$$

For larger values, $x>x_{O}$, of the distance, $x$, of each object from the centre of energy, $m(x)$ will vary in such a manner as to respect the conservation of relativistic energy:

$$
E^{2}=4\left(\left(m+\frac{q^{2}}{4 c^{2} x}\right)^{2} c^{4}+p^{2} c^{2}\right) \equiv 4\left(m(x)^{2} c^{4}+p^{2} c^{2}\right)
$$

Since $E$ is constant, due to the conservative nature of the interaction, it is clear that any change in the momentum of the objects must be accompanied by corresponding changes in their masses. Another example of position-dependent masses is given below in Section 6 where circular Keplerian orbits of a system of two electrically charged objects are discussed.

The structure of this paper is as follows: In the following section the RCED formulae for the electric and magnetic force fields of a uniformly moving electric charge are derived from Coulomb's Law and the 4-vector character of the electromagnetic potential. In Section 3 small angle Rutherford scattering is calculated in different inertial frames, using either RCED force fields or the conventional retarded fields. It is shown that the latter, when used in the initial rest frame of an electron scattered by a heavy charged particle, give a result incompatible with both the known Rutherford scattering formula and the Bethe-Bloch energy-loss relation. In this section it is also shown that the forces predicted by the retarded fields do not, in general, respect the law of conservation of relativistic transverse momentum. In Section 4 the breakdown of Gauss' law for the total flux of the RCED electric force field of a charge in uniform motion is demonstrated. In Section 5 the recent 'torque paradox' of Jackson is resolved by employing the RCED force fields, that, unlike the conventional retarded ones, are consistent with SR. Section 6 presents an analysis of circular Keplerian orbits in RCED. The relativistic generalisation of Kepler's third law for such orbits is derived. In Section 7 the question raised by 19th century astronomers, and recalled by Eddington [19], concerning the possibility of stable orbital motion under the influence of retarded fields, is addressed, for the case of circular orbits in electrically bound systems, and a negative answer is found. The final section is a summary of the results obtained. 


\section{Force fields of a moving charge in relativistic clas- sical electrodynamics}

The expressions for the electric and magnetic fields in RCED found in [4] are here rederived for the simpler case of a uniformly moving charge by exploiting the 4-vector character of the electromagnetic potential, $A$, and assuming Coulomb's law of force between static charges with an instantaneous interaction. The usual covariant definitions ${ }^{2}$ of the electric and magnetic fields ${ }^{3}[20]$ are used:

$$
\begin{aligned}
\vec{E} & =-\vec{\nabla} A_{0}-\frac{1}{c} \frac{\partial \vec{A}}{\partial t} \\
\vec{B} & =\vec{\nabla} \times \vec{A}
\end{aligned}
$$

Denoting by S' (coordinates $t^{\prime}, \vec{x}^{\prime}$ ) the rest frame of an electric charge $q$ and $\mathrm{S}$ (coordinates $t, \vec{x})$ a frame, moving with velocity $-v$ along the $x_{1}^{\prime}$ axis, in which the fields are to be evaluated, then $A^{\prime}=(q / r ; 0,0,0)$ in $\mathrm{S}^{\prime}$. If $X, X^{\prime}$ are space-time points in $\mathrm{S}$ and $\mathrm{S}^{\prime}$ respectively at which the fields are to be evaluated while $X_{q}$ and $X_{q}^{\prime}$ are the corresponding space-time positions of the charge, the quantity $r$ is the invariant space-like interval given in $\mathrm{S}^{\prime} \mathrm{by}^{4}$ :

$$
r^{2}=-\left(X^{\prime}-X_{q}^{\prime}\right)^{2}
$$

In view of the instantaneous nature of the Coulomb interaction: $t^{\prime}=t_{q}^{\prime}$, so that:

$$
r^{2}=\left(\overrightarrow{x^{\prime}}-{\overrightarrow{x^{\prime}}}_{q}\right)^{2}
$$

The Lorentz transformation equations between the frames S' and S:

$$
\begin{aligned}
& A_{1}=\gamma\left(A_{1}^{\prime}+\beta A_{0}^{\prime}\right) \\
& A_{0}=\gamma\left(A_{0}^{\prime}+\beta A_{1}^{\prime}\right)
\end{aligned}
$$

give, remembering the Lorentz-scalar character of $r$ :

$$
A=\left(\frac{\gamma q}{r} ; \frac{\gamma \beta q}{r}, 0,0\right)
$$

Substituting (2.7) into (2.1) and (2.2), and performing the partial differentiations, noting that, for the partial time derivative in $(2.1), \vec{x}$ is held constant while $\vec{x}_{q}$ is allowed to vary, the following expressions for the RCED electric and magnetic force fields are obtained:

$$
\begin{aligned}
\vec{E}(R C E D) & =\frac{j_{0} \vec{r}}{c r^{3}}-\frac{\vec{j}(\vec{r} \cdot \vec{v})}{c^{2} r^{3}} \\
\vec{B}(R C E D) & =\frac{q \gamma(\vec{v} \times \vec{r})}{c r^{3}}=\frac{\vec{j} \times \vec{r}}{c r^{3}}
\end{aligned}
$$

where $j \equiv q u=q(\gamma c ; \gamma v, 0,0)$ and $u$ is the 4 -vector velocity of the charge q. Choosing $\vec{r}$ in the 12 plane and with unit vectors $\hat{\imath}, \hat{\jmath}$ parallel to $\mathrm{O} x_{1}$ and $\mathrm{O} x_{2}$ respectively, (2.8) can be written in the convenient form:

$$
\vec{E}\left(R C E D, S_{e}\right)=\frac{q}{r^{2}}\left(\frac{\hat{\imath} \cos \psi}{\gamma}+\gamma \hat{\jmath} \sin \psi\right)
$$

\footnotetext{
${ }^{2}$ Gaussian electromagnetic units are employed.

${ }^{3}$ These are derived from first principles in [4]

${ }^{4} \mathrm{~A}$ time-like metric is used for 4 -vector products
} 
where $\cos \psi=(\vec{r} \cdot \vec{v}) /|\vec{r}||\vec{v}|$. This is the RCED formula used in the following section.

\section{Small angle Rutherford scattering with instanta- neous or retarded fields}

Rutherford scattering of an electron of charge, $-e$, and mass, $m$, by a nucleus of charge, $Z e$, and mass, $M$, is considered in two frames of reference. The first is the rest frame, $\mathrm{S}_{N}$ (coordinates $\left(t^{\prime} ; x^{\prime}, y^{\prime}, z^{\prime}\right)$ ) of the nucleus (essentially the overall centre-of-mass frame of the two body scattering problem) the second is the rest frame, $\mathrm{S}_{e}$ (coordinates $(t ; x, y, z))$, of the initial electron. As in a similar discussion in [21] a large impact parameter, $b$, is considered so that the geometrical deflection of the electron trajectory in $\mathrm{S}_{N}$ and the change in position of the electron in $\mathrm{S}_{e}$, during the scattering interaction, are neglected. Also neglected are changes in the velocities of the electron or nucleus during the scattering interaction. Geometrical parameters in the frames $\mathrm{S}_{N}$ and $\mathrm{S}_{e}$ are defined in Figs. 1a and $1 \mathrm{~b}$ respectively, while Fig.1c shows the definition of the scattering angle, $\theta$, of the electron momentum vector in the centre-of-mass frame $\mathrm{S}_{C M} \simeq \mathrm{S}_{N}$. The scattering angle is given, in the small angle approximation that is made here, as :

$$
\theta \simeq \frac{\Delta p}{p}
$$

$p$ is the electron momentum in $\mathrm{S}_{C M}$ and $\Delta p$ is calculated by integrating the transverse impulse produced by the electric field of the nucleus during the passage of the electron in $\mathrm{S}_{N}$ or of the nucleus in $\mathrm{S}_{e}$. Small effects due to magnetic fields in $\mathrm{S}_{e}$ (which, since they are transverse to the electron velocity, do not contribute to $|\Delta p|)$ are neglected. The electric field is calculated either from the RCED formula (2.10) or by use of the standard formula for the electric field of a moving charge given in textbooks on classical electrodynamics. This is the same as the 'present time' version of the retarded Liénard-Wiechert (LW)[22] field, which was first derived by Heaviside [23].

In the frame $\mathrm{S}_{N}$, the electric field at the position of the electron is given, in both cases, by a static Coulomb field, since the source charge is at rest:

$$
\vec{E}\left(S_{N}, R C E D\right)=\vec{E}\left(S_{N}, L W\right)=\hat{\imath^{\prime}} E_{L}\left(S_{N}\right)+\hat{\jmath^{\prime}} E_{T}\left(S_{N}\right) \equiv \frac{Z e}{r^{2}}\left(\hat{\imath} \cos \psi+\hat{\jmath^{\prime}} \sin \psi\right)
$$

where $\psi$ is the angle between the vectors $\vec{r}$ and $\vec{v}$ (see Fig.1a) and $\hat{\imath^{\prime}}, \hat{j^{\prime}}$ are unit vectors in the $x^{\prime}$ - and $y^{\prime}$-directions. In the frame $\mathrm{S}_{e}$, where the nucleus is in motion, different formulae are found for the electric field in the two cases:

$$
\begin{aligned}
\vec{E}\left(S_{e}, R C E D\right) & =\frac{Z e}{r^{2}}\left(\frac{\hat{\imath} \cos \psi}{\gamma}+\gamma \hat{\jmath} \sin \psi\right) \\
\vec{E}\left(S_{e}, L W\right) & =\frac{Z e}{r^{2} \gamma^{2}} \frac{(\hat{\imath} \cos \psi+\hat{\jmath} \sin \psi)}{\left(1-\beta^{2} \sin ^{2} \psi\right)^{\frac{3}{2}}}
\end{aligned}
$$

where $\hat{\imath}$ and $\hat{\jmath}$ are unit vectors in the $x$ - and $y$-directions. Although the transverse fields given by (3.3) and (3.4) agree when $\psi=\pi / 2$, the angular dependence for fixed $r$ is very different, as may be seen in Figs.2 and 3. which show respectively $E_{L} r^{2} / Z e$ and $E_{T} r^{2} / Z e$, 
where $E_{L}$ and $E_{T}$ are the longitudinal and transverse components of $\vec{E}$, repectively, as a function of $\psi$, for various values of $\beta$. It can be seen in Fig. 3 that the denominator of the right side of (3.4) strongly damps the strength of the transverse electric field, except for values of $\psi$ close to $\pi / 2$, for values of $\beta$ close to unity. On performing the integral over $\psi$ to calculate $\Delta p$ a much smaller value of $\theta$ is then to be expected for a given value of $b$ for $\vec{E}\left(S_{e}, L W\right)$ as compared to $\vec{E}\left(S_{e}, R C E D\right)$. This is indeed found to be the case.

a)

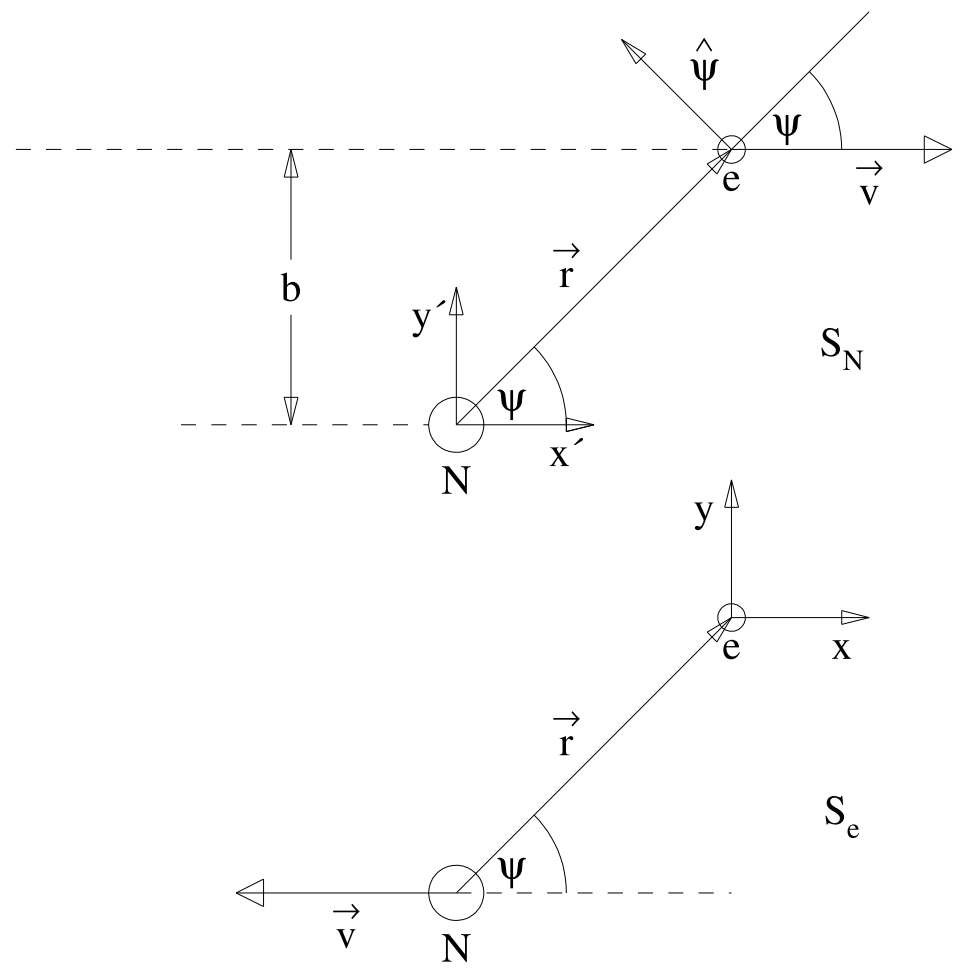

c)

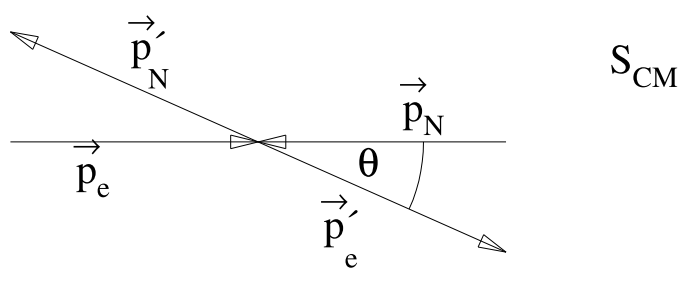

Figure 1: Geometrical parameters for the calculation of Rutherford scattering in different frames. In a) the nucleus, $N$, is at rest in $S_{N}$, and the scattered electron, e, is in motion. In b) the electron is initially at rest in $S_{e}$ while the nucleus is in motion. c) shows the definition of the electron scattering angle, $\theta$, in the overall center-of-mass frame, $S_{C M}$. To a good approximation, $S_{N}$ and $S_{C M}$ are the same. 


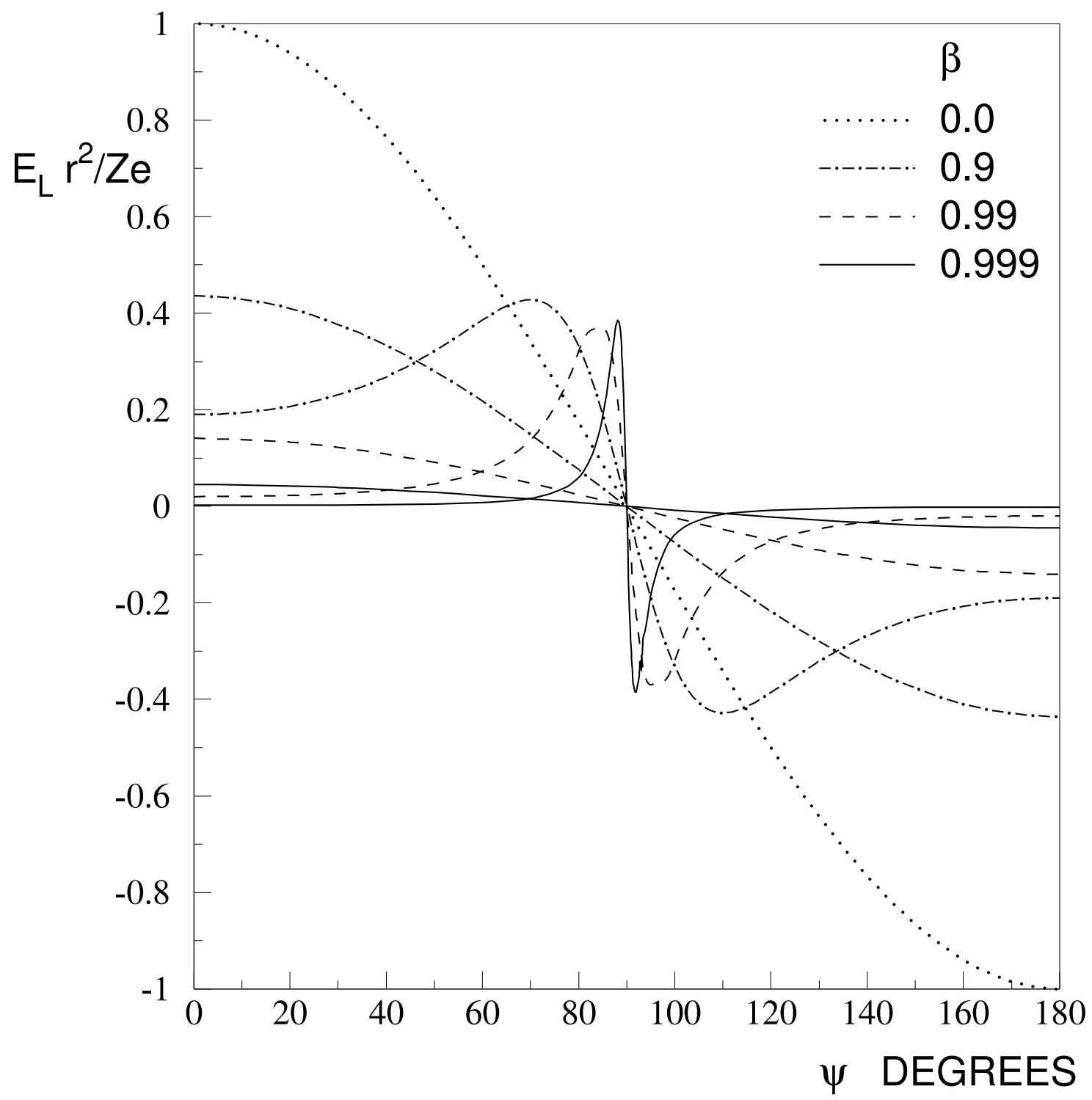

Figure 2: The longitudinal electric field of a uniformly moving electric charge, scaled by the factor $r^{2} / Z e$, as a function of angular position $\psi$, for different relativistic velocities $\beta=v / c$. The slowly varying cosine curves correspond to the RCED formula (3.3), the more rapidly varying ones to the retarded CEM field of (3.4). 


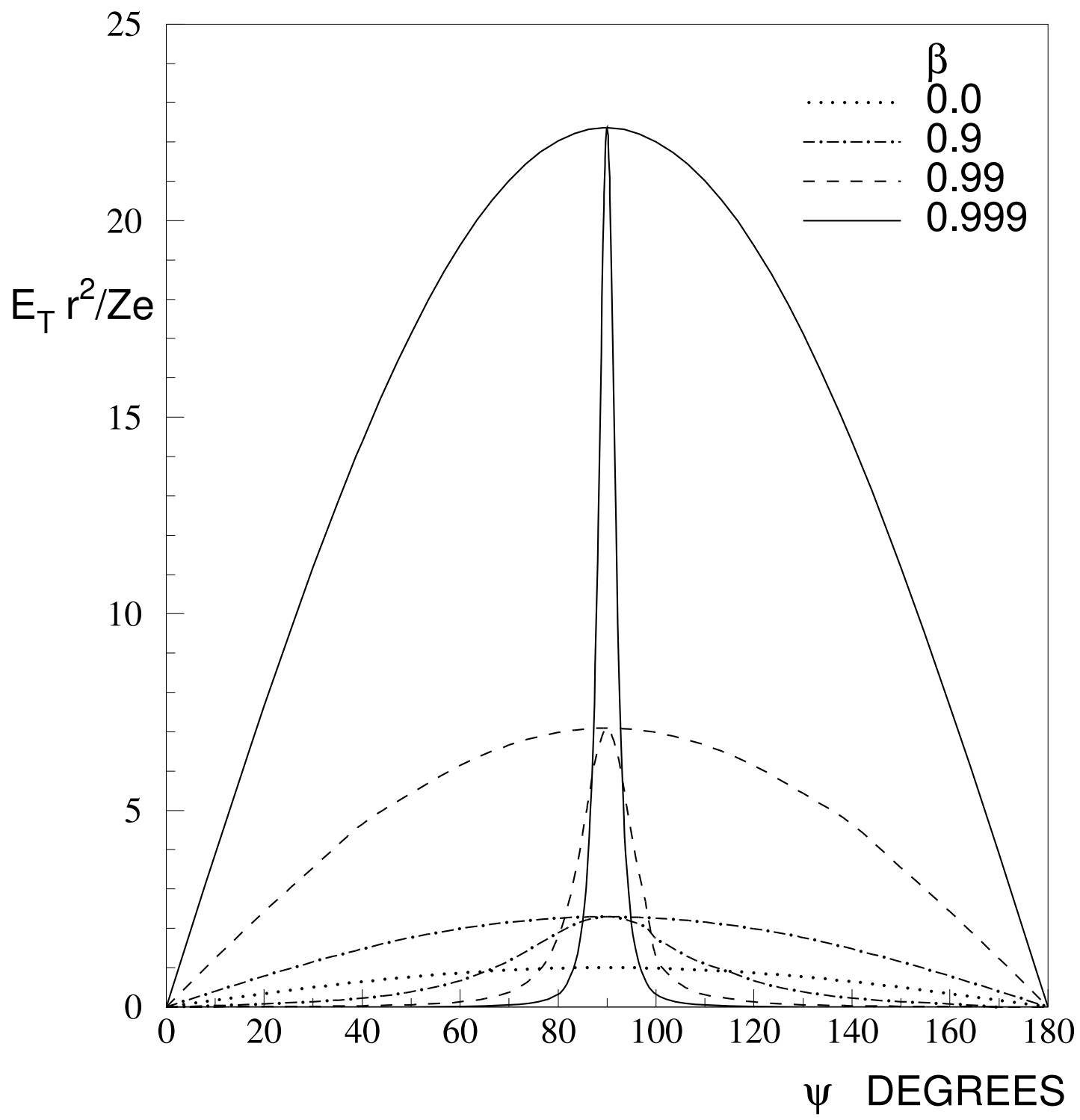

Figure 3: The transverse electric field of a uniformly moving electric charge, scaled by the factor $r^{2} / Z e$, as a function of angular position $\psi$, for different relativistic velocities $\beta=v / c$. The slowly varying sine curves correspond to the RCED formula (3.3), the more rapidly varying ones to the retarded CEM field of (3.4). 
In the frame $\mathrm{S}_{N}$ the transverse impulse ${ }^{5}$ is given, according to (3.2) as:

$$
\Delta p\left(S_{N}\right)=\int_{-\infty}^{\infty} e E_{T}\left(S_{N}\right) d t^{\prime}=Z e^{2} \int_{-\infty}^{\infty} \frac{\sin \psi}{r^{2}} d t^{\prime}
$$

Consideration of the component of the electron velocity $\vec{v}$ perpendicular to $\vec{r}$ in the scattering plane (i.e. in the direction of the unit vector $\hat{\psi}$, see Fig.1a) gives the relation:

$$
v_{\psi}=r \frac{d \psi}{d t^{\prime}}=-v \sin \psi
$$

Multiplying both sides of (3.6) by $\mathrm{r}$ and noting that, from the geometry of Fig.1a, $r \sin \psi=$ $b$ gives, on rearrangement,

$$
d t^{\prime}=-\frac{r^{2} d \psi}{b v}
$$

Using (3.7), (3.5) is converted into an integral over $\psi$ :

$$
\Delta p\left(S_{N}\right)=\frac{Z e^{2}}{b v} \int_{0}^{\pi} \sin \psi d \psi=\frac{2 Z e^{2}}{b v}
$$

(3.1) and (3.8) give, for the relation between the scattering angle and the impact parameter:

$$
\theta\left(S_{N}\right)=\frac{2 Z e^{2}}{p b v}
$$

in agreement, in the small angle limit, with the exact result (i.e. one valid also for $v \simeq c)[21]:$

$$
\tan \frac{\theta}{2}=\frac{Z e^{2}}{m v^{2} b}
$$

Calculating now the transverse impulse in the frame $\mathrm{S}_{e}$ using the RCED formula (3.3) for the electric field gives, instead of (3.5):

$$
\Delta p\left(S_{e}, R C E D\right)=\int_{-\infty}^{\infty} e E_{T}\left(, S_{e}, R C E D\right) d t=Z e^{2} \int_{-\infty}^{\infty} \gamma \frac{\sin \psi}{r^{2}} d t
$$

Relativistic time dilatation gives the relation ${ }^{6}: \gamma d t=d t^{\prime}$. Hence the right sides of (3.5) and (3.11) are identical, leading to the result:

$$
\theta\left(S_{e}, R C E D\right)=\theta\left(S_{N}\right)=\frac{2 Z e^{2}}{p b v}
$$

Using now (3.4) and (3.7) as well as the relation $\gamma d t=d t^{\prime}$, the transverse impulse imparted by the LW electric field in the frame $\mathrm{S}_{e}$ is :

$$
\Delta p\left(S_{e}, L W\right)=\int_{-\infty}^{\infty} e E_{T}\left(S_{e}, L W\right) d t=\frac{Z e^{2}}{\gamma^{3} b v} \int_{0}^{\pi} \frac{\sin \psi d \psi}{\left(1-\beta^{2} \sin ^{2} \psi\right)^{\frac{3}{2}}}=\frac{2 Z e^{2}}{\gamma b v}
$$

(3.13) gives, for the electron scattering angle:

$$
\theta\left(S_{e}, L W\right)=\frac{2 Z e^{2}}{\gamma p b v}
$$

\footnotetext{
${ }^{5} \Delta p$ is treated throughout as a positive quantity.

${ }^{6}$ Note that $t$ is the proper time $\tau$ in the initial electron rest frame, so that $d t^{\prime}=\gamma d \tau$ is the usual time dilatation formula relating time increments in 'stationary' and 'moving' inertial frames.
} 
Thus for a given value of $b$ the value of the scattering angle is reduced by a factor $1 / \gamma$ as compared to the calculation in $\mathrm{S}_{N}$.

In Jackson's book [21] a result consistent with (3.12) was found for the calculation in the frame $\mathrm{S}_{e}$ using $\vec{E}\left(S_{e}, L W\right)^{7}$. This is because the calculation effectively used, in (3.13), the time $t^{\prime}$ defined in the frame $\mathrm{S}_{N}$ instead of the correct time $t$ in the frame $\mathrm{S}_{e}$. Making the replacement $d t \rightarrow d t^{\prime}$ in (3.13) and using (3.7) gives a result, the one quoted by Jackson, consistent with (3.12). A footnote in [21] indicates that the author has realised that $\Delta p$ was calculated in the frame $\mathrm{S}_{e}$, whereas the relation for the scattering angle, (3.1), is valid, instead, in $\mathrm{S}_{C M}$, where the longitudinal electron momentum is $\gamma m v$. There is then the statement: 'The reader may verify that (13.3)' (i.e. (3.9) above)'and (13.4)'(i.e. (3.10) above) 'are also correct in the frame in which the electron is at rest by transforming the angles from the CM system to the laboratory'. Actually, this is impossible to do, because the electron scattering angle is undefined in the electron rest frame. The calculations of $\Delta p$ in the frames $\mathrm{S}_{N}$ and $\mathrm{S}_{e}$ above using the retarded field (3.4) show, instead, results that differ by a factor $\gamma$. The calculation of the scattering angle performed in $\mathrm{S}_{e}$ then gives an angle a factor $\gamma$ smaller than the known Rutherford scattering result (2.10). Use of $\Delta p$ calculated with (3.4) in $\mathrm{S}_{e}$ then leads to a $(1 / \gamma)^{2}$ dependence of the energy-loss formula at relativistic velocities, in contradiction both with the Bethe-Bloch formula and experiment [24].

That the transverse field in (3.3) is larger, by a factor $\gamma$, than the static value in (3.2), for any value of $\psi$, is a necessary consequence of the invariance of relativistic transverse momentum will now be demonstrated. Since the Lorentz transformation leaves invariant transverse spatial intervals: $\Delta y=\Delta y^{\prime}$, relativistic transverse momentum must also be conserved:

$$
p_{T} \equiv m \frac{d y}{d \tau}=m \frac{d y^{\prime}}{d \tau}=m \gamma v=m \gamma^{\prime} v^{\prime}
$$

Considering now the transverse momentum, $p_{T}$, of the electron in Rutherford scattering and associating the frame $\mathrm{S}$ with $\mathrm{S}_{N}$ and $\mathrm{S}^{\prime}$ with $\mathrm{S}_{e}{ }^{8}$, then, since $v^{\prime} \ll v, \gamma^{\prime} \simeq 1$. That is, the motion of the electron in $\mathrm{S}_{e}$ is non-relativistic. If $p_{T}$ is generated by transverse forces $F_{T}, F_{T}^{\prime}$ in $\mathrm{S}_{N}, \mathrm{~S}_{e}$ respectively, conservation of transverse momentum gives:

$$
\Delta p_{T}=F_{T} \Delta t=F_{T}^{\prime} \Delta t^{\prime}
$$

so that

$$
\frac{F_{T}^{\prime}}{F_{T}}=\frac{\Delta t}{\Delta t^{\prime}}=\frac{\gamma}{\gamma^{\prime}} \simeq \gamma
$$

since $\Delta \tau=\Delta t / \gamma=\Delta t^{\prime} / \gamma^{\prime}$. Thus conservation of transverse momentum requires the transverse electric field in $\mathrm{S}_{e}$ to be a factor $\gamma$ larger than that in $\mathrm{S}_{N}$ for all values of $\psi$. This condition is respected by $\vec{E}(R C E D)$ in (3.3), but not by $\vec{E}(L W)$ in (3.4), for which the condition is satisfied only for $\psi=\pi / 2$. The forces generated by the retarded field (3.4) therefore do not, in general, respect the invariance of relativistic transverse momentum, in different inertial frames, that is required by SR.

\footnotetext{
${ }^{7}$ The integration variable was a time rather than the angle $\psi$.

${ }^{8}$ This, the opposite of the previous assignment, is done for clarity of presentation. Here it is the rest and moving frames of the scattered electron rather than those of the nucleus $N$ that are under discussion.
} 


\section{Breakdown of Gauss' theorem for the electric field flux of a moving charge in relativistic classical elec- trodynamics}

The total flux of the electric field generated by a moving charge:

$$
\phi_{E}=\int_{S} \vec{E} \cdot d \vec{S}
$$

is readily calculated from the formulae (3.3) and (3.4) for different choices of a surface, $\mathrm{S}$, that completely surrounds the charge. The results obtained for (i) a spherical surface centered on the charge and (ii) a cylindrical surface with axis parallel to the direction of motion of the charge, of length $2 \ell$ and radius $a$, with the charge at the axis mid-point, are, for the RCED and the retarded LW fields:

Sphere:

$$
\begin{aligned}
\phi_{E}(R C E D) & =\frac{4 \pi Z e}{3}\left(2 \gamma+\frac{1}{\gamma}\right) \\
\phi_{E}(L W) & =4 \pi Z e
\end{aligned}
$$

Cylinder:

$$
\begin{aligned}
\phi_{E}(R C E D) & =4 \pi Z e\left(\left(\gamma-\frac{1}{\gamma}\right) \cos \theta_{0}+\frac{1}{\gamma}\right) \\
\phi_{E}(L W) & =4 \pi Z e
\end{aligned}
$$

where $\tan \theta_{0}=a / \ell$.

The flux of the retarded LW field is independent of the velocity of the charge and of the form of the surface $\mathrm{S}$, but, as can be seen in Fig.3, the electric field strength peaks strongly near $\psi=\pi / 2$ for large velocities $\beta \simeq 1$. The number of field lines is conserved but their spatial distribution changes with the velocity of the charge. This change in the distribution of the field lines is often interpreted as due to relativistic 'length contraction' [25]. However, the flux of the RCED force fields do not, as is clear from (4.2) and (4.4), respect Gauss' theorem, or the electrostatic Maxwell equation, when the source charge is in motion. The flux depends both on the velocity of charge and the geometry of the surface S. For large values of $\gamma$ the flux is proportional to $\gamma$, but with different multiplicative constants for spherical or cylindrical surfaces. In RCED, both the tranverse field and the total flux are proportional to $\gamma$ at large $\gamma$, whereas in the same limit the transverse LW field is proportional to $\gamma$ only for $\psi=\pi / 2$, while the total flux is velocity independent. There should be nothing shocking in these properties of the fields. As discussed in [4], the force fields are only mathematical abstractions introduced in order to write inter-particle forces in a compact manner. The physical reality resides in the particles and the forces between them, not in the fields. All equations of motion may be written down and solved without the necesssity to introduce either the concept of a force or that of a field. Just this approach is taken in the discussion of Keplerian orbits in Section 6 below. The total 
flux of the field, though readily calculable, has no independent physical meaning and it is irrelevant, for the dynamical description of the motion of the charges, whether the flux respects, of not, Gauss' theorem. However the forces derived from the fields must respect on the one hand, the constraints of SR and on other be consistent, in the appropraiate limit, with the known and tested predictions of QED. As the several examples considered in the present paper show, this is the case for the instantaneous RCED fields, but not for the retarded LW ones.

\section{Resolution of Jackson's torque paradox}

Another example of the incompatibility of the LW retarded fields with relativistic invariance is provided by the 'torque paradox' recently pointed out by Jackson [26]. Replace in Fig.1b the moving nucleus by an electron at rest. The electric field at $e$ is then a static Coulomb field parallel to $\vec{r}$. This is the only force-generating field in the frame $\mathrm{S}_{e}$ and gives no torque. Now, on boosting from $\mathrm{S}_{e}$ into $\mathrm{S}_{N}$, both electrons move with velocity $v$ parallel to the x-axis. According to the LW formula (3.4), the electric field is still parallel to $\vec{r}$ and therefore generates no torque. However, in $\mathrm{S}_{N}$, the electric current constituted by the lower electron produces at $e$ a magnetic field of magnitude:

$$
\vec{B}(L W)=\vec{\beta} \times \vec{E}(L W)
$$

The corresponding Lorentz force on $e$ is parallel to Oy and gives a non-vanishing torque, $\vec{T}:$

$$
\vec{T}=-e \vec{r} \times[\vec{\beta} \times(\vec{\beta} \times \vec{E}(L W))]=-\hat{k} e \beta^{2} \sin \psi \cos \psi|\vec{E}(L W)|
$$

where $\hat{k}$ is a unit vector parallel to the $z$-axis ${ }^{9}$. Since this torque does not exist in the inertial frame $S_{e}$, related to $S_{N}$ by Lorentz transformation, there is a manifest breakdown of the relativity principle. This is Jackson's torque paradox. A similar problem was previously pointed out [27] in connection with the interpretation of Trouton and Noble experiment [28].

The RCED fields at $e$ in $\mathrm{S}_{N}$, on replacing $\mathrm{N}$ by an electron moving with velocity $v$ along Ox' are, using (2.9) and (2.10), and dropping the primes on the unit vectors in $\mathrm{S}_{N}$ :

$$
\begin{aligned}
\vec{E}(R C E D) & =-\frac{e}{r^{2}}\left(\frac{\hat{\imath} \cos \psi}{\gamma}+\gamma \hat{\jmath} \sin \psi\right) \\
\vec{B}(R C E D) & =-\frac{e \hat{k}}{r^{2}} \gamma \beta \sin \psi
\end{aligned}
$$

The corresponding Lorentz force, $\vec{F}$, on $e$ is:

$$
\begin{aligned}
\vec{F}(R C E D) & =-e[\vec{E}(R C E D)+\vec{\beta} \times \vec{B}(R C E D)] \\
& =\frac{e^{2}}{r^{2}}\left[\frac{\hat{\imath} \cos \psi}{\gamma}+\gamma \hat{\jmath} \sin \psi+\beta(\hat{\imath} \times \hat{k})(\gamma \beta \sin \psi)\right] \\
& =\frac{e^{2}}{r^{2}}\left[\frac{\hat{\imath} \cos \psi}{\gamma}+\hat{\jmath} \gamma\left(1-\beta^{2}\right) \sin \psi\right]
\end{aligned}
$$

\footnotetext{
${ }^{9}$ This formula is identical to $\operatorname{Eqn}(7)$ of $[26]$ on making the replacements $\theta \rightarrow \psi, \beta_{0} \rightarrow \beta$ and $t=0$ in the latter.
} 


$$
=\frac{e^{2}}{\gamma r^{2}}[\hat{\imath} \cos \psi+\hat{\jmath} \sin \psi]=\frac{e^{2}}{\gamma r^{2}} \vec{r}
$$

Thus the Lorentz force in $\mathrm{S}_{N}$ is radial and, consistent with relativistic invariance, there is no torque in this frame.

\section{Circular Keplerian orbits in Relativistic Classical Electrodynamics}

In [4] equations of motion, of two mutually interacting charged objects $\mathrm{O}_{1}$ and $\mathrm{O}_{2}$, were derived. They may be most simply written in the 'fieldless' and 'forceless' form:

$$
\begin{aligned}
& \frac{d \overrightarrow{p_{1}}}{d t}=m_{1} \frac{d\left(\gamma_{1} \overrightarrow{v_{1}}\right)}{d t}=\frac{q_{1}}{c}\left[\frac{j_{2}^{0} \vec{r}+\overrightarrow{\beta_{1}} \times\left(\overrightarrow{j_{2}} \times \vec{r}\right)}{r^{3}}-\frac{1}{c r} \frac{d \overrightarrow{j_{2}}}{d t}-\overrightarrow{j_{2}} \frac{\left(\vec{r} \cdot \overrightarrow{\beta_{2}}\right)}{r^{3}}\right] \\
& \frac{d \overrightarrow{p_{2}}}{d t}=m_{2} \frac{d\left(\gamma_{2} \overrightarrow{v_{2}}\right)}{d t}=-\frac{q_{2}}{c}\left[\frac{j_{1}^{0} \vec{r}+\overrightarrow{\beta_{2}} \times\left(\overrightarrow{j_{1}} \times \vec{r}\right)}{r^{3}}+\frac{1}{c r} \frac{d \overrightarrow{j_{1}}}{d t}-\overrightarrow{j_{1}} \frac{\left(\vec{r} \cdot \overrightarrow{\beta_{1}}\right)}{r^{3}}\right]
\end{aligned}
$$

where $m_{1}$ and $m_{2}$ are the masses of the objects in the absence of mutual interaction, and $q_{1}$ and $q_{2}$ their electric charges. The vector $\vec{r} \equiv \overrightarrow{r_{12}} \equiv \overrightarrow{r_{1}}-\overrightarrow{r_{2}}$ gives the spatial separation of the objects in their centre-of-mass frame. The 4-vector current $j_{i}$ of the object $\mathrm{O}_{i}$ is defined as $j_{i} \equiv q_{i} u_{i}$, where $u_{i}$ is the 4 -vector velocity $u_{i} \equiv\left(c \gamma_{i} ; c \gamma_{i} \vec{\beta}_{i}\right)$. In the following, the motion of the objects in their centre-of-mass frame is considered, and the origin of the vectors $\overrightarrow{r_{1}}$ and $\overrightarrow{r_{2}}$ is the common centre of energy, O, of the objects.

Because of the relation connecting the velocity, current and energy-momentum 4vectors:

$$
u=\frac{j}{q}=\frac{p}{m}
$$

the differential equations (6.1) and (6.2) are coupled via the $d \vec{j} / d t$ terms on their right sides. Solving these equations for the time derivatives of the relativistic velocities of the two objects gives the equations:

$$
\begin{aligned}
\frac{d\left(\gamma_{1} \vec{v}_{1}\right)}{d t}= & \frac{q_{1} q_{2}}{m_{1}\left[1-\frac{\left(q_{1} q_{2}\right)^{2}}{m_{1} m_{2} c^{4} r^{2}}\right]} \frac{1}{r^{3}} \times \\
& \left\{\gamma_{2}\left[\vec{r}+\vec{\beta}_{1} \times\left(\vec{\beta}_{2} \times \vec{r}\right)-\overrightarrow{\beta_{2}}\left(\vec{r} \cdot \vec{\beta}_{2}\right)\right]\right. \\
& \left.+\frac{q_{1} q_{2} \gamma_{1}}{m_{2} c^{2} r}\left[\vec{r}+\vec{\beta}_{2} \times\left(\overrightarrow{\beta_{1}} \times \vec{r}\right)-\vec{\beta}_{1}\left(\vec{r} \cdot \vec{\beta}_{1}\right)\right]\right\} \\
\frac{d\left(\gamma_{2} \overrightarrow{v_{2}}\right)}{d t}= & \frac{-q_{1} q_{2}}{m_{1}\left[1-\frac{\left(q_{1} q_{2}\right)^{2}}{m_{1} m_{2} c^{4} r^{2}}\right]} \frac{1}{r^{3}} \times \\
& \left\{\gamma_{1}\left[\vec{r}+\vec{\beta}_{2} \times\left(\vec{\beta}_{1} \times \vec{r}\right)-\overrightarrow{\beta_{1}}\left(\vec{r} \cdot \vec{\beta}_{1}\right)\right]\right. \\
& \left.+\frac{q_{1} q_{2} \gamma_{2}}{m_{1} c^{2} r}\left[\vec{r}+\vec{\beta}_{1} \times\left(\overrightarrow{\beta_{2}} \times \vec{r}\right)-\vec{\beta}_{2}\left(\vec{r} \cdot \vec{\beta}_{2}\right)\right]\right\}
\end{aligned}
$$

The differential geometry of uniform circular motion is illustrated in Fig.4. The vectors $\vec{v}_{1}$ or $\vec{v}_{2}$ are perpendicular to $\vec{r}$ at all times. Also $\left|\vec{v}_{1}\right|,\left|\vec{v}_{2}\right|, r_{1}$ and $r_{2}$ are constant at all 
times. It follows from the geometry of Fig.4. that, to first order in $\delta r, \delta v$ :

$$
\frac{\delta \phi}{\delta t}=\frac{1}{v_{1}} \frac{\left|\delta \vec{v}_{1}\right|}{\delta t}=\frac{1}{v_{2}} \frac{\left|\delta \vec{v}_{2}\right|}{\delta t}=\frac{1}{r_{1}} \frac{\left|\delta \vec{r}_{1}\right|}{\delta t}=\frac{v_{1}}{r_{1}}=\frac{1}{r_{2}} \frac{\left|\delta \vec{r}_{2}\right|}{\delta t}=\frac{v_{2}}{r_{2}}
$$

For the case of uniform circular motion, $q_{1}$ and $q_{2}$ must have opposite signs and (6.4) and (6.5) simplify to:

$$
\begin{aligned}
\gamma_{1} \frac{d \vec{v}_{1}}{d t} & =\frac{-\left|q_{1}\right|\left|q_{2}\right|\left(1+\beta_{1} \beta_{2}\right)}{m_{1}\left[1-\frac{\left(q_{1} q_{2}\right)^{2}}{m_{1} m_{2} c^{4} r^{2}}\right]}\left[\gamma_{2}-\frac{\left|q_{1}\right|\left|q_{2}\right| \gamma_{1}}{m_{2} c^{2} r}\right] \frac{\vec{r}}{r^{3}} \\
\gamma_{2} \frac{d \vec{v}_{2}}{d t} & =\frac{\left|q_{1}\right|\left|q_{2}\right|\left(1+\beta_{1} \beta_{2}\right)}{m_{2}\left[1-\frac{\left(q_{1} q_{2}\right)^{2}}{m_{1} m_{2} c^{4} r^{2}}\right]}\left[\gamma_{1}-\frac{\left|q_{1}\right|\left|q_{2}\right| \gamma_{2}}{m_{2} c^{2} r}\right] \frac{\vec{r}}{r^{3}}
\end{aligned}
$$

These equations may be combined to yield the relation:

$$
\mathcal{E}_{1}^{*} \frac{d \vec{v}_{1}}{d t}+\mathcal{E}_{2}^{*} \frac{d \vec{v}_{2}}{d t}=0
$$

where

$$
\begin{aligned}
\mathcal{E}_{1}^{*} & \equiv \frac{\gamma_{1} m_{1}}{\gamma_{2}-\frac{\left|q_{1}\right|\left|q_{2}\right| \gamma_{1}}{m_{2} c^{2} r}} \\
\mathcal{E}_{2}^{*} & \equiv \frac{\gamma_{2} m_{2}}{\gamma_{1}-\frac{\left|q_{1}\right|\left|q_{2}\right| \gamma_{2}}{m_{1} c^{2} r}}
\end{aligned}
$$

Since, for uniform circular motion, $\mathcal{E}_{1}^{*}$ and $\mathcal{E}_{2}^{*}$ are constant, (6.9) may also be written as:

$$
\frac{d}{d t}\left[\mathcal{E}_{1}^{*} \vec{v}_{1}+\mathcal{E}_{2}^{*} \vec{v}_{2}\right]=\frac{d}{d t}\left[\overrightarrow{p_{1}^{*}}+\overrightarrow{p_{2}^{*}}\right]=0
$$

or, equivalently,

$$
\frac{d \overrightarrow{p_{1}^{*}}}{d t}=-\frac{d \overrightarrow{p_{2}^{*}}}{d t}
$$

where $\overrightarrow{p_{i}^{*}} \equiv \mathcal{E}_{i}^{*} \vec{v}_{i}, i=1,2$. This is just the expression of Newton's third law for the two mutually interacting objects.

Equations (6.10) and (6.11) then suggest that the electromagnetic interaction between the objects modifies their masses as a function of their velocites and spatial separation according to the relations:

$$
\begin{aligned}
m_{1}^{*} & \equiv \frac{m_{1}}{\gamma_{2}-\frac{\left|q_{1}\right|\left|q_{2}\right| \gamma_{1}}{m_{2} c^{2} r}} \\
m_{2}^{*} & \equiv \frac{m_{2}}{\gamma_{1}-\frac{\left|q_{1}\right|\left|q_{2}\right| \gamma_{2}}{m_{1} c^{2} r}}
\end{aligned}
$$

Thus, when proper account is taken of the modification of the masses of the objects, due to their mutual electromagnetic interaction, there is not, in the case considered here, any breakdown of Newton's third law.

Combining (6.6) and (6.9)

$$
\frac{\left|d \vec{v}_{1}\right| / d t}{\left|d \vec{v}_{2}\right| / d t}=\frac{\mathcal{E}_{2}^{*}}{\mathcal{E}_{1}^{*}}=\frac{v_{1}}{v_{2}}=\frac{r_{1}}{r_{2}}
$$




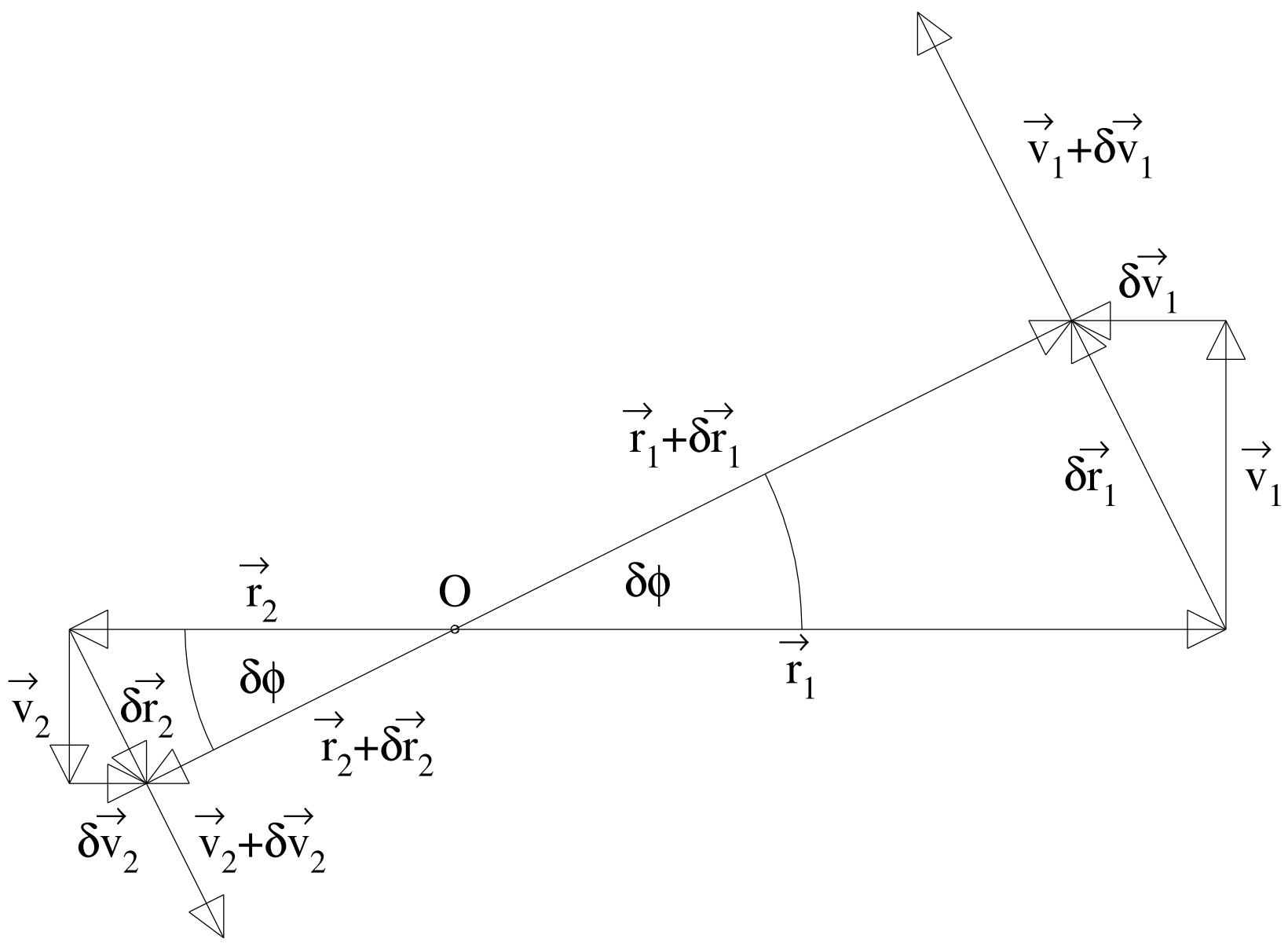

Figure 4: Differential geometry of the position and velocity vectors of two objects, $O_{i}$, $(i=1,2)$ executing uniform circular motion about their common center-of-energy $O$. As $\delta \vec{r}_{i}$ and $\delta \vec{v}_{i} \rightarrow 0, \delta \vec{r}_{i} \perp \vec{r}_{i}, \delta \vec{v}_{i} \perp \vec{v}_{i},\left|\vec{r}_{i}\right|=$ const. and $\left|\vec{v}_{i}\right|=$ const. 


$$
\begin{aligned}
& \mathcal{E}_{2}^{*} v_{2}=\mathcal{E}_{1}^{*} v_{1} \\
& \mathcal{E}_{2}^{*} r_{2}=\mathcal{E}_{1}^{*} r_{1}
\end{aligned}
$$

Equation(6.17), which may be written as $\left|\overrightarrow{p_{2}^{*}}\right|=\left|\overrightarrow{p_{1}^{*}}\right|$, is consistent with momentum conservation in the overall centre-of-mass frame: $\overrightarrow{p_{2}^{*}}=-\overrightarrow{p_{1}^{*}}$, while (6.18) states that the origins of $\vec{r}_{1}$ and $\vec{r}_{2}$ are at the centre of energy of the two interacting objects.

The period of rotation, $\tau$, of either object in its circular Keplerian orbit, is readily derived from (6.7) or (6.8) and the geometrical relation (6.6). Considering the object $\mathrm{O}_{1}$ :

$$
\gamma_{1} \frac{\left|d \overrightarrow{v_{1}}\right|}{d t}=\frac{\left|q_{1}\right|\left|q_{2}\right|\left(1+\beta_{1} \beta_{2}\right)}{m_{1} \gamma_{1}\left[1-\frac{\left(q_{1} q_{2}\right)^{2}}{m_{1} m_{2} c^{4} r^{2}}\right]}\left[\gamma_{2}-\frac{\left|q_{1}\right|\left|q_{2}\right| \gamma_{1}}{m_{2} c^{2} r}\right] \frac{1}{r^{2}}=v_{1} \frac{d \phi}{d t}=\frac{2 \pi r_{1}}{\tau} \frac{2 \pi}{\tau}
$$

Using the relations:

$$
r=r_{1}+r_{2}=r_{1}\left(1+\frac{r_{2}}{r_{1}}\right)=r_{1}\left(1+\frac{\mathcal{E}_{1}^{*}}{\mathcal{E}_{2}^{*}}\right)=\frac{r_{1} \mathcal{E}_{1}^{*}}{\mathcal{E}^{*}}
$$

where $\mathcal{E}^{*}$ is the 'reduced energy':

$$
\mathcal{E}^{*} \equiv \frac{\mathcal{E}_{1}^{*} \mathcal{E}_{2}^{*}}{\mathcal{E}_{1}^{*}+\mathcal{E}_{2}^{*}}
$$

to eliminate $r_{1}$ in favour of $r$ on the right side of (6.19) yields a formula relating the rotation period of the objects to their spatial separation:

$$
\begin{aligned}
\tau^{2} & =\frac{(2 \pi)^{2} m_{1} \gamma_{1} \mathcal{E}^{*}\left[1-\frac{\left(q_{1} q_{2}\right)^{2}}{m_{1} m_{2} c^{4} r^{2}}\right] r^{3}}{\mathcal{E}_{1}^{*}\left|q_{1}\right|\left|q_{2}\right|\left(1+\beta_{1} \beta_{2}\right)\left[\gamma_{2}-\frac{\left|q_{1} \| q_{2}\right| \gamma_{1}}{m_{2} c^{2} r}\right]} \\
& =\frac{(2 \pi)^{2} \mathcal{E}^{*}\left[1-\frac{\left(q_{1} q_{2}\right)^{2}}{m_{1} m_{2} c^{4} r^{2}}\right] r^{3}}{\left|q_{1}\right|\left|q_{2}\right|\left(1+\beta_{1} \beta_{2}\right)}
\end{aligned}
$$

where, in the last member of (6.22), (6.10) has been used. This is the relativistic generalisation of Kepler's third law. The usual classical result is recovered in the limit $c \rightarrow \infty$. Note that no kinematial approximation whatever has been made in the derivation. Equation (6.22) gives the exact result for the period of a circular orbit to all orders in $\left|q_{1}\right|\left|q_{2}\right| /\left(m_{1} c^{2} r\right),\left|q_{1}\right|\left|q_{2}\right| /\left(m_{2} c^{2} r\right), \beta_{1}$ and $\beta_{2}$.

\section{The impossibility of uniform circular motion under retarded Liéard-Wiechert fields}

Several years before the advent of special relativity the effect on electric and magnetic fields, propagated at the speed of light, was considered by Liénard and Wiechert [22]. A good explanation of the physics arguments presented by these authors may be found in Reference [8]. In this case the values of the fields at a given space-time point are given by the sum of the contributions from all sources that lie on the backward three-dimensional light-cone of the space-time point considered. Neglecting relativistic effects (as of course 
Liénard and Wiechert were obliged to do) the instantaneous classical scalar and vector potentials $\phi(X)=q / r$ and $\vec{A}(X)=q \vec{v} / r$ are found to be modified according to the relations $[8]$ :

$$
\begin{aligned}
& \phi_{r e t}(X)=\left\{\frac{q}{\left(r-\frac{\vec{v} \cdot \vec{r}}{c}\right)}\right\}_{t_{r e t}} \\
& \vec{A}_{r e t}(X)=\left\{\frac{q \vec{v}}{c\left(r-\frac{\vec{v} \cdot \vec{r}}{c}\right)}\right\}_{t_{r e t}}
\end{aligned}
$$

where the quantities in large curly brackets are evaluated at the retarded time: $t_{r e t}=$ $t-r / c$ at which the source charge is sampled by a signal moving along the backward light-cone of the space-time point $X=(c t ; \vec{x})$. The electric and magnetic fields derived from these potentials are given by the expressions[29]:

$$
\begin{aligned}
& \vec{E}_{\text {ret }}^{\text {vel }}(X)=\left\{\frac{q\left(1-\beta^{2}\right)(\vec{r}-\vec{\beta} r)}{(r-\vec{\beta} \cdot \vec{r})^{3}}\right\}_{t_{\text {ret }}} \\
& \vec{E}_{\text {ret }}^{a c c}(X)=\left\{\frac{q \vec{r} \times[(\vec{r}-\vec{\beta} r) \times d \vec{\beta} / d t]}{c(r-\vec{\beta} \cdot \vec{r})^{3}}\right\}_{t_{r e t}} \\
& \vec{B}_{\text {ret }}(X)=-\left(\vec{E}_{\text {ret }}^{\text {vel }}(x)+\vec{E}_{\text {ret }}^{a c c}(x)\right) \times\left\{\begin{array}{l}
\vec{r} \\
r
\end{array}\right\}_{t_{\text {ret }}}
\end{aligned}
$$

$\vec{E}_{\text {ret }}^{v e l}(X)$ and $\vec{E}_{\text {ret }}^{a c c}(X)$ give the contributions to the electric field due to the velocity and acceleration respectively of the source charge.

The possibility of uniform circular motion of two objects with equal and opposite electric charges and different masses, interacting mutually via the fields (7.3)-(7.5) above is now investigated. As in the previous section, the geometrical constraints of uniform circular motion are imposed and a solution of the equations of motion of the objects consistent with these constraints is sought. Various geometrical parameters used to describe the system are defined in Fig.5. The objects $\mathrm{O}_{1}$ and $\mathrm{O}_{2}$ of masses, in the absence of interaction, $m_{1}$ and $m_{2}$ and electric charges $q$ and $-q$ are assumed to perform uniform circular motion about their centre of energy $\mathrm{O}$. The radii of the circular orbits of $\mathrm{O}_{1}$ and $\mathrm{O}_{2}$ are $r_{1}$ and $r_{2}$. Cartesian axes $\mathrm{Ox}$, Oy and $\mathrm{Oz}$ specified by unit vectors $\hat{\imath}, \hat{\jmath}$ and $\hat{k}$ are chosen with y-axis parallel to $\vec{r}_{2}$ at some instant and x-axis in the plane of the orbits. The resultant force on $\mathrm{O}_{1}$ due to the retarded fields of $\mathrm{O}_{2}$ is now calculated and examined for consistency with uniform circular motion. According to $\operatorname{Eqn}(7.3) \vec{E}_{\text {ret }}^{v e l}\left(X_{1}\right) \equiv \vec{E}_{\text {ret }}^{v e l}(1)$ points to the "present position' (assuming uniform motion) of the source charge. Calculating the retarded fields of $\mathrm{O}_{2}$ at $\mathrm{O}_{1}$ when the latter is at position $\mathrm{A}$ in Fig.5 at $t=0$, implies that the fields are given by the formulae (7.3)-(7.5) when $\mathrm{O}_{2}$ was at position $\mathrm{B}$ at time $t=-r / c . \vec{E}_{r e t}^{\text {vel }}(1)$ points towards the point $\mathrm{C}$ on the tangent to the orbit of $\mathrm{O}_{2}$ at $\mathrm{B}$. The distance $\mathrm{BC}$ is $r \beta_{2}{ }^{10}$. The calculation of the retarded fields from the Eqns(7.3)-(7.5) and the geometry

\footnotetext{
${ }^{10}$ The object $\mathrm{O}_{2}$ does not actually follow this trajectory under its assumed circular orbit. However, causality implies that only the velocity of the source at the instant it is sampled on the backward lightcone of the point at which the field is evaluated is relevant. Any change in the motion (velocity or acceleration) of the source at later times therefore cannot affect the retarded field. The retarded field $\vec{E}_{r e t}^{v e l}(1)$ at $\mathrm{A}$ is therefore the same whether $\mathrm{O}_{2}$ follows a circular trajectory BE or the straight one BC.
} 


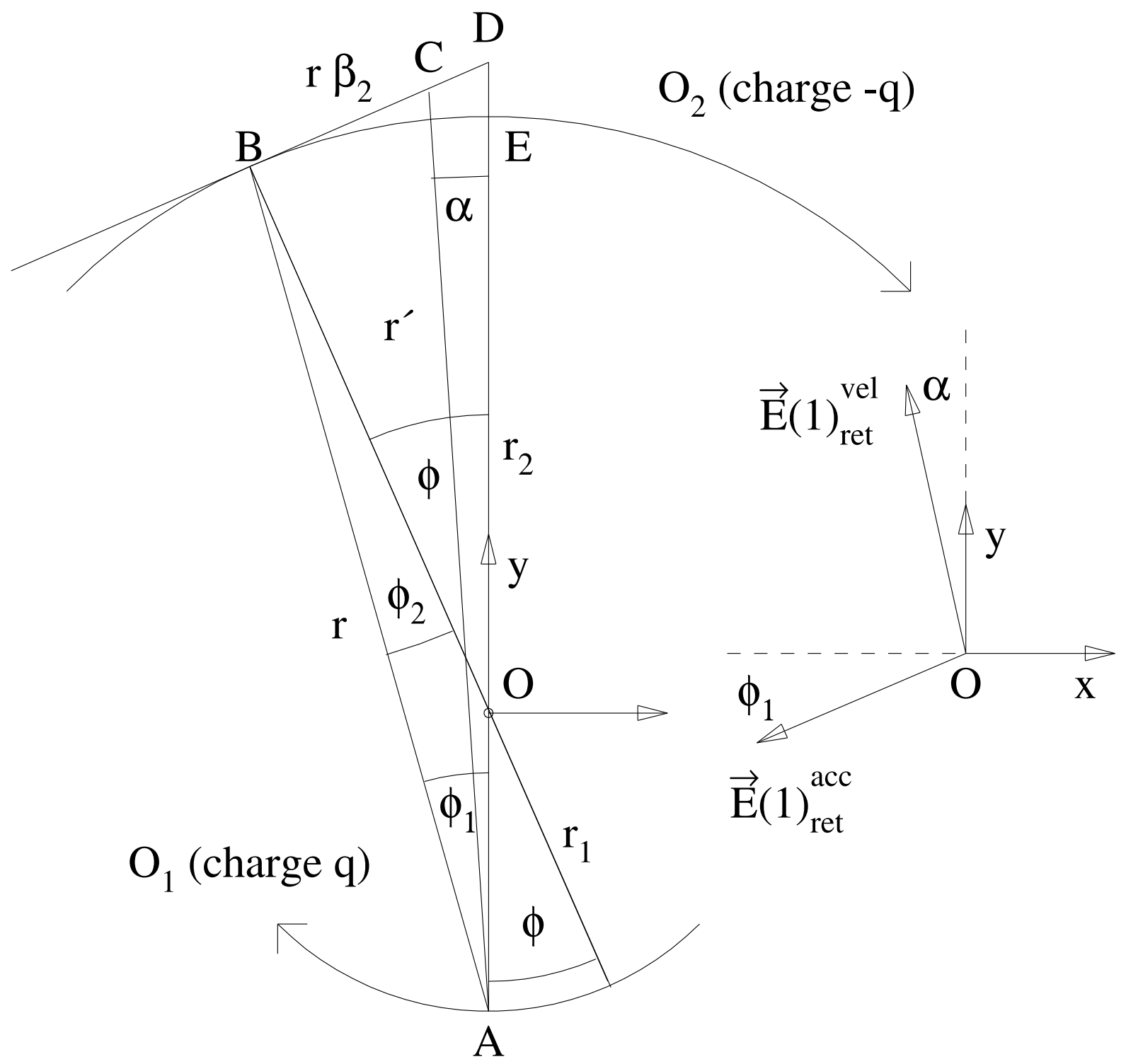

Figure 5: Geometry of uniform circular motion of two oppositely charged objects, $O_{1}$ and $\mathrm{O}_{2}$, about their common centre of energy $O$. The forces on $\mathrm{O}_{1}$ at $A$ generated by the retarded fields (7.3)-(7.5) of $\mathrm{O}_{2}$ are calculated. The force fields are produced by $\mathrm{O}_{2}$ at the position $B$. Various geometrical parameters are defined. The directions of the electric fields at $A$ associated with the velocity and acceleration of $\mathrm{O}_{2}$ are shown in the inset figure. 
of Fig.5 is presented in the Appendix. The following results are obtained:

$$
\begin{aligned}
& \vec{E}_{\text {ret }}^{\text {vel }}(1)=-\frac{\hat{\imath} q \beta_{2}^{3}}{3 r_{2}^{2}}+\frac{\hat{\jmath} q\left[1-\beta_{2}\left(2 \beta_{1}+\beta_{2} / 2\right)\right]}{\left(r_{1}+r_{2}\right)^{2}}+O\left(\beta_{2}^{4}\right) \\
& \vec{E}_{\text {ret }}^{a c c}(1)=-\frac{\hat{\imath} q \beta_{2}^{2}\left(\beta_{1}+\beta_{2}\right)}{r_{2}\left(r_{1}+r_{2}\right)}+O\left(\beta_{2}^{4}\right) \\
& \vec{B}_{\text {ret }}(1)=-\frac{\hat{k} q \beta_{1}}{\left(r_{1}+r_{2}\right)^{2}}+O\left(\beta_{1}^{3}\right)
\end{aligned}
$$

According to the Lorentz force equation, the total force acting on $\mathrm{O}_{1}$ is:

$$
\vec{F}_{r e t}(1)=-\frac{\hat{\imath} q^{2} \beta_{2}^{2}}{r_{2}}\left[\frac{\beta_{1}+\beta_{2}}{r_{1}+r_{2}}+\frac{\beta_{2}}{3 r_{2}}\right]+\frac{\hat{\jmath} q^{2}}{r_{1}+r_{2}}\left[\frac{1-\beta_{2}\left(2 \beta_{1}+\beta_{2} / 2\right)}{r_{1}+r_{2}}\right]+O\left(\beta_{2}^{4}\right)
$$

By symmetry, the force on $\mathrm{O}_{2}$ due to the retarded fields of $\mathrm{O}_{1}$ is:

$$
\vec{F}_{r e t}(2)=\frac{\hat{\imath} q^{2} \beta_{1}^{2}}{r_{1}}\left[\frac{\beta_{1}+\beta_{2}}{r_{1}+r_{2}}+\frac{\beta_{1}}{3 r_{1}}\right]-\frac{\hat{\jmath} q^{2}}{r_{1}+r_{2}}\left[\frac{1-\beta_{1}\left(2 \beta_{2}+\beta_{1} / 2\right)}{r_{1}+r_{2}}\right]+O\left(\beta_{1}^{4}\right)
$$

Inspection of (7.9) and (7.10) demonstrates the impossiblity of uniform circular motion under the action of retarded Liénard - Wiechert fields. There is a manifest breakdown of Newton's third law, that cannot, due to the non-factorisable force components on the right sides of (7.9) and (7.10), be restored, as in the discussion of the previous section, by the introduction of different effective masses for the interacting objects. Also, since the resultant force is non-central, an unbalanced torque acts on the system. The unbalanced force is:

$$
\begin{aligned}
\vec{F}_{r e t}(1)+\vec{F}_{r e t}(2) & =-\hat{\imath} q^{2}\left[\frac{\beta_{1}+\beta_{2}}{r_{1}+r_{2}}\left(\frac{\beta_{2}^{2}}{r_{2}}-\frac{\beta_{1}^{2}}{r_{1}}\right)+\frac{1}{3}\left(\frac{\beta_{2}^{3}}{r_{2}^{2}}-\frac{\beta_{1}^{3}}{r_{1}^{2}}\right)\right] \\
& +\frac{\hat{\jmath} q^{2}\left(\beta_{1}^{2}-\beta_{2}^{2}\right)}{2\left(r_{1}+r_{2}\right)^{2}}+O\left(\beta_{1}^{4}, \beta_{2}^{4}\right)
\end{aligned}
$$

The clockwise (accelerating) torque is of magnitude:

$$
\Gamma=q^{2} \beta_{1} \beta_{2}\left[\frac{2\left(\beta_{1}+\beta_{2}\right)}{r_{1}+r_{2}}+\frac{1}{3}\left(\frac{\beta_{2}}{r_{2}}+\frac{\beta_{1}}{r_{1}}\right)\right]+O\left(\beta_{1}^{6}, \beta_{2}^{6}\right)
$$

In [19] Eddington discussed the case of the (almost circular) orbits of Jupiter and the Sun under the action of retarded gravitational fields. A simple geometrical argument suggests that Jupiter and the Sun would then be subject to accelerating torques. Eddington then considered the analogous electromagnetic problem, and citing the Heaviside formula (3.4) for the electric field of a moving charge, stated that the direction of the retarded force is 'very nearly' in the direction of the centre of mass in this case. It was then concluded that there is no unbalanced torque problem for the Sun-Jupiter system, and that it may be generally assumed that the gravitational force propagates at the speed of light. The calculation presented above shows this conclusion to be incorrect. As the formulae (6.1) and (6.2) follow from only the inverse-square force law, SR and Hamilton's Principle, similar formula, with the relacement $q_{1} q_{2} \rightarrow-G m_{1} m_{2}$, are expected to hold in Newtonian gravitation, when special relativistic corrections are included. In this case also, stable circular orbits cannot be obtained with retarded gravitational fields. 
A calculation considering the stability of circular orbits for the case of gravitational forces has been previously published [30]. As for the electromagnetic forces just considered, and for similar geometrical reasons, an accelerating torque was found in the case of retarded gravitational fields. By comparing the predicted rate of change of their periods with precision data on the rate of change of the observed periods of binary pulsars, a lower limit of $2800 \mathrm{c}$ for the speed of propagation of gravitational force fields was set. The conclusions of Ref. [30] were questioned in Refs. [31, 32]. However, the argumentation of these authors was based on an analogy between retarded gravitational and electric force fields. They concluded that since the retarded LW electric field of Eqn(3.4) is radial at the 'present time' there is no torque. As discussed above, a similar remark was made by Eddington [19]. This conclusion is refuted by the calculation presented above. The present-time retarded electric field is radial only for a source charge in straight-line motion. As the above calculation and the analogous gravitational one of Ref. [30] show, the force is no longer radial when the circular geometry of the orbits is properly taken into account. Carlip's argument in Ref. [31] has also been rebutted in Ref. [33].

\section{Summary}

The present paper is a sequel to [4] where inter-charge forces in classical electrodynamics were derived from Coulomb's law, SR and Hamilton's Principle. An essential aspect of the derivation is the instantaneous nature of the electromagnetic forces, demonstrated in [4] to be a prediction of QED. In standard text books on classical electromagnetism $[8,9,10]$ no clear distinction is made between fields describing mechanical forces (the effect, in QED, of the exchange of space-like virtual photons) and fields giving a classical description of radiative processes (in QED, the creation or destruction of real, on-shell, photons). The importance of such a distinction, the former fields being instantaneous, the latter propagating at the speed of light, was pointed out many years ago by Chubykalo and Smirnov-Rueda [34]. This conjecture is fully supported by the work presented in [4] and the present paper.

The distinction between the standard text-book formulae, denoted in the present paper by the labels CEM or LW (for Liénard-Wiechert) and those (denoted by RCED) derived in [4] or Section 2 above, is most succinctly expressed in terms of the electromagnetic potentials generated by uniformly moving electric charges in the different theories. In CEM, no distinction is made between fields describing mechanical forces and radiation processes. In both cases the electromagnetic potential is assumed to be of the form first derived by Liénard and Wiechert [22]:

$$
\begin{aligned}
A^{0}(C E M) & =\left\{\frac{q}{\left(r-\frac{\vec{v} \cdot \vec{r}}{c}\right)}\right\}_{t_{r e t}} \\
\vec{A}(C E M) & =\left\{\frac{q \vec{v}}{\left(r-\frac{\vec{v} \cdot \vec{r}}{c}\right)}\right\}_{t_{r e t}}
\end{aligned}
$$

where all quantities in the large curly brackets are evaluated at the retarded time $t_{\text {ret }}=$ $t-r / c$. In RCED there are two physically distinct types of fields. The first, describing 
mechanical inter-charge forces are derived, using (2.1) and (2.2), from the potentials:

$$
\begin{aligned}
& A_{f o r}^{0}(R C E D)=\frac{q \gamma}{r}=\frac{q u^{0}}{c r} \\
& \vec{A}_{f o r}(R C E D)=\frac{q \gamma \vec{v}}{c r}=\frac{q \vec{u}}{c r}
\end{aligned}
$$

where $u \equiv(\gamma c: \gamma \vec{v})$ is the 4-vector velocity of the moving charge. These potentials as well as the associated fields and forces are instantaneous. The retarded potentials of RCED, describing radiation, are the same as (8.3) and (8.4) above, except for a retarded time argument. The different physical interpretations of the fields derived from $A_{\text {for }}$ and the retarded potential are discussed in [4]. See also [34]. It is perhaps surprising, 100 years after the advent of SR, that the formulae (8.1) and (8.2) are still the generally accepted ones for the electromagnetic potential of a moving charge. Indeed $A^{0}$ and $\vec{A}$ in these equations are manifestly not components of a 4 -vector, and so it is not to be expected that the fields and forces derived from them will respect SR. It has been demonstrated in the present paper that indeed they do not, whereas the RCED fields and forces do.

In fact since (8.1) and (8.2) were derived by Liénard and Wiechert some seven years before Einstein's first SR paper, it would be little short of miraculous if they constituted a 4-vector. A similar remark applies to the consistency with SR of the 'present time' formula (3.4) for the retarded electric field of a uniformly moving charge. This may be derived from (8.1) and (8.2), but was first obtained by Heaviside [23] in 1888. It may be noted, on the other hand, that a velocity-dependent scalar potential, consistent with (8.3) up to $\mathrm{O}\left(\beta^{2}\right)$ was proposed as early as 1861 by Riemann [35 $]^{11}$.

The mechanical forces in CEM derived from (8.1) and (8.2) have been compared above with those of RCED derived from (8.3) and (8.4). In Section 3 it is shown that use of the CEM formula (3.4) to calculate small-angle Rutherford scattering gives a scattering angle smaller by a factor $1 / \gamma$ than the well-known standard result, that is recovered by use of the RCED formula (3.3). It is also shown in Section 3 that that conservation of relativistic transverse momentum requires that transverse forces in different inertial frames scale according to $1 / \gamma(\operatorname{see} \operatorname{Eqn}(3.17))$. This condition is respected by the RCED fields but not by the CEM ones. In Section 4 it is shown that the flux of the electric field of a moving charge in RCED does not (unlike in CEM where the electric flux is the same for a charge at rest or in motion) respect Gauss' Law. The flux depends both on the velocity of the charge and the geometry of the bounding surface. The electric field is a mathematical abstraction useful to simplify dynamical formulae expressed in terms of forces, but has, unlike charges and their motion, no objective existence. In Section 5, Jackson's 'torque paradox' [26] for two interacting charges, where electric and magnetic fields are derived from the CEM potentials (8.1) and (8.2) is discussed. A non-vanishing torque is predicted in one inertial frame, a vanishing one in another, in contradiction with the special relativity principle. It is shown that use of RCED fields gives a vanishing

\footnotetext{
${ }^{11}$ The same Bernhard Riemann (1826-1866) is better known for his hypothesis concerning the distribution of prime numbers, for pioneering applications of topology to the theory of complex functions and, with Lobatchevsky, the invention of non-Euclidean geometry. Together with Weber and Clausius, he attempted to construct an action-at-a-distance theory of electromagnetic forces in which the fundamental physical objects were charged particles, not fields. See the second reference in [35]. This is also the approach adopted in [4] by the present author. Such theories were generally superseded in the second half of the 19th century by Maxwell's electromagnetic field theory.
} 
torque in all inertial frames, consistent with SR. In Section 6 it is demonstrated that stable, circular, Keplerian orbits are possible for two oppositely-charged objects using exact RCED dynamical formulae. An exact relativistic generalisation of Kepler's third law relating the orbit period to the separation of the charges is derived for this case. In Section 7 the impossiblity of stable circular motion under forces derived from retarded CEM fields is demonstrated. Such forces do not respect Newton's third law and, as already conjectured during the 19th Century for the analogous gravitational case [19] and recently demonstrated by explicit calculation [30], generate an unbalanced torque. Stable orbital motions of electrons in atoms or of planets around the Sun are only possible if (as Newton assumed for the case of gravity) the inverse-square-law electrical or gravitational forces act instantaneously. 


\section{Appendix}

Denoting by $\delta t$ the time of passage of $\mathrm{O}_{2}$ from $\mathrm{B}$ to $\mathrm{E}$ (Fig.5) then:

$$
\delta t=\frac{r}{c}=\frac{\phi r_{2}}{v_{2}}
$$

From the geometry of Fig.5:

$$
\begin{aligned}
r & =r_{1} \cos \phi_{1}+r_{2} \cos \phi_{2} \\
r_{1} \sin \phi_{1} & =r_{2} \sin \phi_{2} \\
\phi & =\phi_{1}+\phi_{2} \\
r_{1} v_{2} & =r_{2} v_{1}
\end{aligned}
$$

It follows from (A1-A5) that: $\phi=\beta_{1}+\beta_{2}, \phi_{1}=\beta_{2}+\mathrm{O}\left(\phi_{1}^{3}\right)$ and $\phi_{2}=\beta_{1}+\mathrm{O}\left(\phi_{2}^{3}\right)$.

Making the small angle approximations:

$$
\sin \phi \simeq \phi-\frac{\phi^{3}}{6} \quad \cos \phi \simeq=1-\frac{\phi^{2}}{2}
$$

equations (A2)-(A5) may be combined to give:

$$
\begin{aligned}
r & \simeq\left(r_{1}+r_{2}\right)\left(1-\frac{r_{1} \beta_{2}^{2}}{2 r_{2}}\right) \\
& =\left(r_{1}+r_{2}\right)\left(1-\frac{\beta_{1} \beta_{2}}{2}\right)+O\left(\beta_{2}^{3}\right) \\
r-\overrightarrow{\beta_{2}} \cdot \vec{r} & =r\left(1+\beta_{2} \sin \phi_{2}\right) \\
& \simeq r\left(1+\beta_{1} \beta_{2}\right) \\
& =\left(r_{1}+r_{2}\right)\left(1+\frac{\beta_{1} \beta_{2}}{2}\right)+O\left(\beta_{2}^{3}\right)
\end{aligned}
$$

also

$$
\vec{r}^{\prime} \equiv \vec{r}-\overrightarrow{\beta_{2}} r=r^{\prime}(\hat{\imath} \sin \alpha-\hat{\jmath} \cos \alpha)
$$

Substituting (A6)-(A8) into (7.3) and noting that $q_{2}=-q$ and $r^{\prime}=r \sec \left(\phi_{1}-\alpha\right)$ gives:

$$
\begin{aligned}
\vec{E}_{r e t}^{v e l}(1) & \simeq \frac{q\left(1-\beta_{2}^{2}\right)(-\hat{\imath} \sin \alpha+\hat{\jmath} \cos \alpha)}{r^{2}\left(1+\beta_{1} \beta_{2}\right)^{3} \cos \left(\phi_{1}-\alpha\right)} \\
& \simeq \frac{q\left(1-\beta_{2}^{2}\right)\left(1+\frac{\beta_{2}^{2}}{2}\right)(-\hat{\imath} \sin \alpha+\hat{\jmath} \cos \alpha)}{\left(r_{1}+r_{2}\right)^{2}\left(1+2 \beta_{1} \beta_{2}\right)} \\
& =\frac{q\left[-\hat{\imath} \alpha+\hat{\jmath}\left\{1-\beta_{2}\left(2 \beta_{1}+\beta_{2} / 2\right)\right\}\right]}{\left(r_{1}+r_{2}\right)^{2}}+O\left(\beta_{1}^{4}, \beta_{2}^{4}\right)
\end{aligned}
$$

The last member follows since, as shown below, $\alpha$ is of order $\beta_{2}^{3}$. 
From the geometry of Fig.5:

$$
\begin{aligned}
\tan \alpha & =\frac{C D \cos \phi}{r_{1}+r_{2} \sec \phi-C D \sin \phi} \\
& =\frac{\left(r_{2} \tan \phi-r \beta_{2}\right) \cos \phi}{r_{1}+r_{2} \sec \phi-\left(r_{2} \tan \phi-r \beta_{2}\right) \sin \phi} \\
& =\frac{r_{2}(\sin \phi-\phi \cos \phi)}{r_{1}+r_{2} \sec \phi-r_{2}(\tan \phi-\phi) \sin \phi}
\end{aligned}
$$

where (A1) has been used. Noting that

$$
\begin{aligned}
\sin \phi-\phi \cos \phi & =\phi-\frac{\phi^{3}}{6}-\phi+\frac{\phi^{3}}{2}+\ldots=\frac{\phi^{3}}{6}+O\left(\phi^{5}\right) \\
\tan \phi \sin \phi-\phi \sin \phi= & \frac{\left(\phi-\frac{\phi^{3}}{6}\right)^{2}}{1-\frac{\phi^{2}}{2}}-\phi^{2}+\frac{\phi^{4}}{6}+\ldots=\frac{\phi^{4}}{3}+O\left(\phi^{6}\right) \\
& \sec \phi=1+\frac{\phi^{2}}{2}+\ldots
\end{aligned}
$$

(A10) gives the result:

$$
\alpha \simeq \arctan \left[\left(\frac{r_{2}}{r_{1}+r_{2}}\right) \frac{\phi^{3}}{3}\right]=\left(\frac{r_{1}+r_{2}}{r_{2}}\right)^{2} \frac{\beta_{2}^{3}}{3}+O\left(\beta_{2}^{5}\right)
$$

Substitution of $\alpha$ given by (A11) into (A9) gives Eqn(7.6) of the text.

Since the modulus of the velocity vector of an object in uniform circular motion is constant, the increment $\delta \overrightarrow{\beta_{2}}$ for $\mathrm{O}_{2}$ is anti-parallel to the radius vector $\vec{r}_{2}$. It then follows from Fig.5 and the geometrical conditions (6.6) that:

$$
\vec{r} \times\left[\left(\vec{r}-\overrightarrow{\beta_{2}} r\right) \times d \overrightarrow{\beta_{2}} / d t\right]=\frac{r r^{\prime} \beta_{2} v_{2} \sin \phi}{r_{2}}\left[\hat{\imath} \cos \phi_{1}+\hat{\jmath} \sin \phi_{1}\right]+O\left(\beta_{2}^{4}\right)
$$

Subsituting (A12) and (A7) into (7.4) gives:

$$
\begin{aligned}
\vec{E}_{r e t}^{a c c}(1) & \simeq-\frac{q \beta_{2}^{2}\left(\beta_{1}+\beta_{2}\right)\left[\hat{\imath} \cos \phi_{1}+\hat{\jmath} \sin \phi_{1}\right]}{r r_{2}\left(1+\beta_{1} \beta_{2}\right)^{3} \cos \left(\phi_{1}-\alpha\right)} \\
& \simeq-\frac{q \beta_{2}^{2}\left(1+\frac{\beta_{2}^{2}}{2}\right)\left(\beta_{1}+\beta_{2}\right)\left[\hat{\imath}\left(1-\frac{\beta_{2}^{2}}{2}\right)+\hat{\jmath} \beta_{2}\right]}{\left(r_{1}+r_{2}\right) r_{2}\left(1+\beta_{1} \beta_{2}\right)^{3}\left(1-\frac{\beta_{1} \beta_{2}}{2}\right)}
\end{aligned}
$$

which yields Eqn(7.7) of the text on retaining only terms up to $\beta_{2}^{3}, \beta_{2}^{2} \beta_{1}$. 


\section{References}

[1] R.P.Feynman, R.B.Leighton and M.Sands, 'The Feynman Lectures in Physics' (Addison-Wesley, Reading Massachusetts, 1963), Vol 1 Ch 28-1, 'Electromagnetism I' Ch 12-7.

[2] See for example the reviews in: T.Kinoshita, 'Quantum Elecrodynamics', (World Scientific, Singapore 1990).

[3] R.P.Feynman, 'QED The Strange Theory of Light and Matter', (Princeton University Press, 1985) Chapter 3.

[4] J.H.Field, Phys. Scr. 74702 (2006), http://xxx.lanl.gov/abs/physics/0501130.

[5] A.L.Kholmetskii et al. J. Appl. Phys. $101 \quad 023532 \quad$ (2007). http://xxx.lanl.gov/abs/physics/0601084.

[6] J.H.Field, 'Quantum electrodynamics and experiment demonstrate the non-retarded nature of electrodynamical force fields', http://xxx.lanl.gov/abs/0706.1661.

[7] H.Hertz, Ann. der Physik XXXIV 551 (1888).

[8] W.H.Panofsky and M.Phillips, 'Classical Electricity and Magnetism', 2nd Edition (Addison-Wesley, Cambridge Mass, 1962) Ch 19.

[9] L.D.Landau and E.M.Lifshitz 'Classical Theory of Fields', Translated by M.Hamermesh, (Pergamon Press, Oxford, 1962).

[10] J.D.Jackson, 'Classical Electrodynamics', Second Edition (John Wiley and Sons, New York, 1975).

[11] R.H.Romer, Am. J. Phys. 34772 (1966).

[12] W.Shockley and R.P.James, Phys. Rev. Lett. 18876 (1967).

[13] S.Coleman and J.H. Van Vleck, Phys. Rev. 1711370 (1968).

[14] W.H.Furry, Am. J. Phys. 37621 (1969).

[15] G.G.Lombardi, Am. J. Phys. 51213 (1983).

[16] V.Hnizdo, Am. J. Phys. 51213 (1983).

[17] O.D.Jefimenko, Eur. J. Phys. 2039 (1999).

[18] See, for example, B.I.Bleaney and B.Bleaney 'Electricity and Magnetism' (O.U.P., Oxford, 1957) Chapter XIX, or C.Kittel, 'Introduction to Solid State Physics', 6th Edition (J.Wiley and Sons New York 1986) Chapter 7.

[19] A.S.Eddington, 'Space, Time and Gravitation, An Outline of General Relativity', (CUP, Cambridge, 1920) Chapter VI.

[20] A.Einstein, Ann. Phys. (Leipzig) 49767 (1916). 
[21] Reference [10] above, Chapter 13, P619.

[22] A.Liénard, L’Eclairage Electrique, 16 pp5, 53, 106 (1898);

E.Wiechert, Archives Néland (2) 5459 (1900).

[23] O.Heaviside, The Electrician, 221477 (1888), Philos. Mag. 272324 (1889).

[24] Review by D.E.Groom and S.R.Klein in: 'Review of Particle Properties', S.Eidelman et al, Phys. Lett. 592242 (2004).

[25] Reference [10] above, Section 11.10.

[26] J.D.Jackson, Am. J. Phys. 721484 (2004).

[27] Ref. [8] above. Section 15.2, P274.

[28] F.T.Trouton and H.R.Noble, Phil. Trans. bf A202 165 (1903), Proc. Roy. Soc. 72 $132(1903)$.

[29] Reference [9], Chapter 8, Section 63, Equations (63.8-9).

[30] T.Van Flandern, Phys. Lett. A250 1 (1998).

[31] S.Carlip, Phys. Lett. A267 81 (2000).

[32] M.Ibison, H.E.Puthoff and S.R.Little, 'The speed of gravity revisited' http://xxx.lanl.gov/abs/physics/9910050.

[33] T.Van Flandern and J.P.Vigier, Foundations of Physics 321031 (2002).

[34] A.E.Chubykalo and R.Smirnov-Rueda, Phys. Rev. E53 5373 (1996).

[35] B.Riemann, Lectures 'Schwere, Elekricitat und Magnetismus', Hannover, 1875, P326. See also E.T.Whittaker, 'A History of Theories of Aether and Electricity', (Am. Inst. Phys., New York, 1987) Chapter 7. 